\title{
Transmission Coefficient and Heat Conduction of a Harmonic Chain with Random Masses: Asymptotic Estimates on Products of Random Matrices
}

\author{
Theo Verheggen \\ Department of Mathematics, Rutgers University, New Brunswick, New Jersey 08903, USA
}

\begin{abstract}
We find upper and lower bounds for the transmission coefficient of a chain of random masses. Using these bounds we show that the heat conduction in such a chain does not obey Fourier's law: For different temperatures at the ends of a chain containing $N$ particles the energy flux falls off like $N^{-1 / 2}$ rather than $N^{-1}$.
\end{abstract}

\section{Introduction}

We study the transmission coefficient and the heat flux of an isotopically disordered harmonic chain. Since the work we shall describe relates much to earlier work $[1, \ldots, 6]$ we shall content ourselves with giving only a brief sketch of the problem before describing the results.

The system consists of a harmonic chain of $N$ particles with random masses. The system is at either end coupled to a semi-infinite chain of identical particles with unit mass (cf. Rubin and Greer [2]). These left and right parts of the chain are described at $t=0$ by Gibbs equilibrium measures (for the semi-infinite system) at temperatures $T_{L}$ and $T_{R}$, respectively. They act as heatbaths for the particles of the system, at sites 0 to $N-1$, whose masses are denoted by $m_{j}, 0 \leqq j \leqq N-1$. The coupling between the particles is nearest neighbor, harmonic, non-random, and the same for all particles.

For a system where there is a harmonic restoring force at every lattice point it was shown by Spohn and Lebowitz [7] that starting from an arbitrary initial state (ensemble density) $\mu_{s}(0)$ for the middle $N$ particles, constituting the system, the measure describing the whole infinite chain and therefore also $\mu_{s}(t)$ approaches, as $t \rightarrow \infty$, a stationary state $\bar{\mu}_{s}$ in which there is a steady heat flux $J(N)$ through the system. Their proof requires some restriction on the masses, but in any case for our system the last statement follows from the explicit calculations in [2] and [3]. For a different system it was shown in [1]. In the system studied in [1] the particles at each end of the chain are coupled to heat baths modelled by white noise whose

* Research supported by a ZWO fellowship and in part by U.S.A.F.O.S.R. grant no. 78-3522 
covariance is proportional to the temperature of the bath and a Langevin damping term.

When the masses $m_{j}, 0 \leqq j \leqq N-1$, are arranged in a regular manner, i.e., $m_{j}$ is periodic in $j$ with period $n$ as in a perfect crystal with $n$ particles per unit cell, then the steady state heat flux $J(N)$ has a well defined non-vanishing limit $J(\infty)$ as $N \rightarrow \infty$. Hence if we write $J(N)=\kappa(N) \frac{T_{L}-T_{R}}{N}$, where $\kappa(N)$ is the "pseudo" heat conductivity, then $\kappa(N) \sim N$ as $N \rightarrow \infty$, and Fourier's law which would correspond to $\kappa(N) \rightarrow \kappa, 0<\kappa<\infty$, does not hold for these systems $[1,3]$.

The situation is quite different, however, if the system is disordered, i.e., if the masses are random. In this case it was proven [1,3] that, as $N \rightarrow \infty, J(N) \rightarrow 0$ for almost all mass distributions, that is $\kappa(N) / N \rightarrow 0$. The question remaining then is the precise behavior of $\kappa(N)$ as $N \rightarrow \infty$. For the system of [1], perturbation type of computations by Matsuda and Ishii [8] and Visscher [17] gave for the average heat conductivity $\langle\kappa(N)\rangle \sim N^{1 / 2}$ resp. $N^{-1 / 2}$, depending on the boundary conditions. These calculations while certainly not rigorous agree also with the results of computer simulations [9, 17, 18]. For our system a rigorous lower bound $\langle\kappa(N)\rangle \geqq C N^{1 / 2}$ was obtained in [2], and computer simulations showed $\langle\kappa(N)\rangle$ $\sim N^{1 / 2}$ [17]. Consequently it was shown by Papanicolau et al. [10] that for the analogous continuous model, a line with a random index of refraction, for $0 \leqq x \leqq L, \kappa(L) \sim L^{1 / 2}$. It is the purpose of this note to prove the result $\kappa(N) \sim N^{1 / 2}$ for the chain with random masses. This will be done in the next sections following a precise formulation of the problem. Since the results involve essentially getting estimates on the norm of a product of random matrices, this question is discussed briefly in the last section.

We assume that the random masses are identically distributed, independent random variables, with probability distribution $P_{m}(d m)$ which is absolutely continuous with respect to Lebesgue measure. For the density $\mu(m)$ we assume that $\mu(m) \neq 0$ only if $\infty>m^{(1)}>m>m^{(0)}>0$.

If $\hat{u}_{j}$ is the displacement from equilibrium of the $j$ 'th particle, then the equation of motion is

$$
m_{j} \ddot{\hat{u}}_{j}+2 \hat{u}_{j}-\hat{u}_{j-1}-\hat{u}_{j+1}=0 .
$$

For a plane wave with frequency $\omega$ travelling through the system

$$
\hat{u}_{j}=u_{j} e^{-i \omega t}
$$

the $u_{j}$ satisfy the relations

$$
-m_{j} \omega^{2} u_{j}+2 u_{j}-u_{j-1}-u_{j+1}=0 \text {. }
$$

Rubin and Greer [2], and O'Connor and Lebowitz [3] related the steady state energy flow $J(N)$ in this model to the transmission coefficient $T(N, \omega)$. For a wave incident from the right $T(N, \omega)$ is determined by the equation of motion and the boundary conditions

$$
\begin{aligned}
\hat{u}_{j}(t) & =D e^{-i(\omega t+k j)}+R e^{-i(\omega t-k j)} & & j \geqq N-1, \\
\hat{u}_{j}(t) & =e^{-i(\omega t+k j)} & & j \leqq 0, \\
\omega & =\omega(k)=2 \sin k / 2 & & 0 \leqq k \leqq \pi .
\end{aligned}
$$


The transmission coefficient is $T(N, \omega)=1 / D$. Then we have, with Boltzmann's constant equal to one,

$$
\langle J(N)\rangle=(4 \pi)^{-1}\left(T_{L}-T_{R}\right) \int_{0}^{2} d \omega\left\langle T^{*} T(N, \omega)\right\rangle .
$$

Our intention is to show the existence of positive constants $J_{-}$and $J_{+}$such that

$$
\frac{T_{L}-T_{R}}{4 \pi} \frac{J_{-}}{N^{1 / 2}} \leqq\langle J(N)\rangle \leqq \frac{T_{L}-T_{R}}{4 \pi} \frac{J_{+}}{N^{1 / 2}}, \quad \text { as } \quad N \rightarrow \infty .
$$

Actually one can prove the existence of

$$
\lim _{N \rightarrow \infty} N^{1 / 2}\langle J(N)\rangle
$$

and calculate it explicitly, Papanicolau [11]. Since (1.4) already implies non-regular heatflow, we will not do this here. Also it may be possible to use our techniques for more complicated problems, like the heat conduction in a strip, where it may not be possible to actually calculate the limit. For the sake of completeness, we mention the result for the case where $\left\langle m_{i}\right\rangle=1$ :

$$
\lim _{N \rightarrow \infty} N^{1 / 2}\langle J(N)\rangle=\frac{T_{L}-T_{R}}{2 \pi^{1 / 2}\left\langle(m-1)^{2}\right\rangle^{1 / 2}} \int_{0}^{\infty} \frac{t \sinh (\pi t) d t}{\left(t^{2}+\frac{1}{4}\right)^{1 / 2} \cosh ^{2} \pi t} .
$$

\section{Statement of the Main Estimates}

In this section we state the main estimates, Corollaries 1 and 2, and show how these imply our main result (1.4).

Corollary 1. There exists a constant $c$ and a function $g(\omega)$, independent of $N$, such that for all $N$ and $0 \leqq \omega \leqq 2$

$$
\left\langle T^{*} T(N, \omega)\right\rangle \leqq c e^{-g(\omega) N}
$$

$g(\omega)$ is continuous, positive for $\omega>0$, and

$$
\lim _{\omega \downarrow 0} g(\omega) / \omega^{2}=g_{0}>0 .
$$

Corollary 2. There exists an integrable function $f\left(\omega^{\prime}\right), 0 \leqq \omega^{\prime}<\infty$, with a positive integral such that

$$
\lim _{\overline{N \rightarrow \infty}}\left\langle T^{*} T\left(N, \frac{\omega^{\prime}}{N^{1 / 2}}\right)\right\rangle \geqq f\left(\omega^{\prime}\right) .
$$

The first to consider this type of estimates were Pastur and Feldman [4] for a continuous case. In that case the details for a rigorous proof were given by Papanicolau [6]. In the next sections we give a proof of the corollaries, now we show how they imply (1.4). In fact for Corollary 1 we only have to prove the statement for $\omega<\omega_{0}$ where $\omega_{0}$ is any positive number because the rest is covered by O'Connor's proof. In the next sections we will prove Corollary 1 for $\omega<\omega_{0}$ where $\omega_{0}$ is the largest frequency for which there are propagating waves, i.e., for which with probability greater than zero $2-m \omega^{2}>-2$. 
Corollary 1 says that for large $N$ the energy transported is in a frequency band $\left[0, N^{-1 / 2}\right]$.

$$
\begin{aligned}
\sqrt{N} \int_{0}^{2}\left\langle T^{*} T(N, \omega)\right\rangle d \omega & =\int_{0}^{2 N^{1 / 2}}\left\langle T^{*} T\left(N, \frac{\omega^{\prime}}{N^{1 / 2}}\right)\right\rangle d \omega^{\prime} \\
& \leqq \int_{0}^{2 N^{1 / 2}} e^{-g_{1} \omega^{\prime 2}} d \omega^{\prime}<J_{+}<\infty
\end{aligned}
$$

Here $g_{1}$ is the positive minimum of $g(\omega) / \omega^{2}$ for $0 \leqq \omega \leqq 2$.

Corollary 2 implies that the contribution of the frequency band $\left[0, N^{-1 / 2}\right]$ is nonzero. By Fatou's lemma

$$
\begin{aligned}
\lim _{N \rightarrow \infty} N^{1 / 2} \int_{0}^{2}\left\langle T^{*} T(N, \omega)\right\rangle d \omega & \geqq \int_{0}^{\infty} \frac{\lim }{N \rightarrow \infty}\left\langle T^{*} T\left(N, \frac{\omega^{\prime}}{N^{1 / 2}}\right)\right\rangle d \omega^{\prime} \\
& \geqq \int_{0}^{\infty} f\left(\omega^{\prime}\right) d \omega^{\prime}=J_{-}>0 .
\end{aligned}
$$

\section{Proof of the Corollaries}

First we transform the 2-point boundary value problem (1.2) into an initial value problem. Equation (1.1) can be written in transfer matrix form

$$
\left[\begin{array}{c}
u_{j+1} \\
u_{j}
\end{array}\right]=\left[\begin{array}{cr}
2-m_{j} \omega^{2} & -1 \\
1 & 0
\end{array}\right]\left[\begin{array}{c}
u_{j} \\
u_{j-1}
\end{array}\right]=S_{j}\left[\begin{array}{c}
u_{j} \\
u_{j-1}
\end{array}\right]
$$

Define two solutions $w_{j}$ and $z_{j}$ by

$$
\begin{array}{ll}
w_{0}=1 / \sqrt{2} & w_{-1}=1 / \sqrt{2} \\
z_{0}=1 / \sqrt{2} & z_{-1}=-1 / \sqrt{2} .
\end{array}
$$

Then it is just a matter of some messy algebra to prove that

$$
\begin{aligned}
T^{*} T(N, \omega)= & 4 \sin ^{2} k\left\{2 \sin ^{2} k+\sin ^{2} k\left(\frac{w_{N}+w_{N-1}}{\sqrt{2}}\right)^{2}\right. \\
& +(1+\cos k)^{2}\left(\frac{w_{N}-w_{N-1}}{\sqrt{2}}\right)^{2}+\sin ^{2} k\left(\frac{z_{N}-z_{N-1}}{\sqrt{2}}\right)^{2} \\
& \left.+(1-\cos k)^{2}\left(\frac{z_{N}+z_{N-1}}{\sqrt{2}}\right)^{2}\right\}^{-1} .
\end{aligned}
$$

Next we introduce for $\omega \neq 0$ polar coordinates by

$$
\begin{aligned}
& \frac{w_{j+1}+w_{j}}{\sqrt{2}}=r_{j+1} \cos \frac{\phi_{j+1}}{2} \\
& \frac{w_{j+1}-w_{j}}{\sqrt{2}}=\frac{1}{2} \omega\langle m\rangle^{1 / 2} r_{j+1} \sin \frac{\phi_{j+1}}{2}
\end{aligned}
$$


and

$$
\begin{aligned}
& \frac{z_{j+1}+z_{j}}{\sqrt{2}}=\frac{2}{\omega\langle m\rangle^{1 / 2}} q_{j+1} \cos \frac{\theta_{j+1}}{2} \\
& \frac{z_{j+1}-z_{j}}{\sqrt{2}}=q_{j+1} \sin \frac{\theta_{j+1}}{2}
\end{aligned}
$$

with

$$
0 \leqq \phi_{j}<4 \pi, \quad 0 \leqq \theta_{j}<4 \pi .
$$

We also define

$$
\varrho_{j}=\frac{1}{j} \log r_{j}, \quad \eta_{j}=\frac{1}{j} \log q_{j} .
$$

The initial values for the polar coordinates are

$$
\phi_{0}=0, r_{0}=1, \quad \theta_{0}=\pi, q_{0}=1 \text {. }
$$

These coordinates may seem peculiar because of the factors $\frac{\langle m\rangle^{1 / 2} \omega}{2}$. Indeed at this stage a simpler choice would do as well. But later we will need this representation.

Again it is a matter of simple algebra to prove the following estimates

$$
\begin{aligned}
& T^{*} T \leqq \frac{4}{2+\alpha_{1} q_{N}^{2}} \quad \text { for } \quad 0<k \leqq \pi \\
& T^{*} T \geqq \frac{4}{2+\alpha_{2} r_{N}^{2}+\alpha_{2} q_{N}^{2}} \quad \text { for } \quad 0<k \leqq \frac{\pi}{2}
\end{aligned}
$$

where

$$
\begin{aligned}
& \alpha_{1}=\min \left(1, \frac{1}{\langle m\rangle}\right) \\
& \alpha_{2}=\max \left(\langle m\rangle, \frac{2}{\langle m\rangle}\right) .
\end{aligned}
$$

Now it is clear that the proof of the theorem depends on knowledge of the asymptotic behavior of $r_{N}$ and $q_{N}$.

We have

$$
\left[\begin{array}{c}
w_{N} \\
w_{N-1}
\end{array}\right]=S_{N-1} \ldots S_{0}\left[\begin{array}{c}
w_{0} \\
w_{1}
\end{array}\right],\left[\begin{array}{c}
z_{N} \\
z_{N-1}
\end{array}\right]=S_{N-1} \ldots S_{0}\left[\begin{array}{c}
z_{0} \\
z_{-1}
\end{array}\right] .
$$

Matsuda and Ishii [8], and Yoshioka [12] were the first to observe that a theorem by Furstenberg [13] applies to these products of random matrices. The theorem states that the norm of the vectors grows exponentially with $N$. This theorem has 
also been used by Casher and Lebowitz [1], O'Connor and Lebowitz [3], and O'Connor [5]. We state their results without proof in Theorem 1. Theorem 1 and the next theorem on large deviations, which we will prove in the next section, are the basis of the proofs.

Theorem 1. With probability one

$$
\begin{aligned}
& \lim _{N \rightarrow \infty} \varrho_{N}=I(\omega) . \\
& \lim _{N \rightarrow \infty} \eta_{N}=I(\omega) .
\end{aligned}
$$

Here I is a function of $\omega$ only, with the following properties. $I(\omega)$ is strictly positive and continuous for $\omega>0$, and

$$
\lim _{\omega \downarrow 0} \frac{I(\omega)}{\omega^{2}}=\frac{1}{8} \frac{\left\langle m^{2}\right\rangle-\langle m\rangle^{2}}{\langle m\rangle}=\frac{1}{8} \tau^{2} .
$$

Theorem 2. There exist a constant $K$ and a function $J(\beta, \omega), \beta \in \mathbb{R}, \omega_{0} \geqq \omega>0$, such that for all $\varepsilon>0, \beta>0, \omega, N>0$

$$
\begin{aligned}
& P\left(\varrho_{N}>I(1+\varepsilon)\right) \leqq K e^{-\beta N I(1+\varepsilon)+J(\beta, \omega) N}, \\
& P\left(\eta_{N}>I(1+\varepsilon)\right) \leqq K e^{-\beta N I(1+\varepsilon)+J(\beta, \omega) N}, \\
& P\left(\eta_{N} \leqq I(1-\varepsilon)\right) \leqq K e^{\beta N I(1+\varepsilon)+J(-\beta, \omega) N} .
\end{aligned}
$$

Here $J(\beta, \omega)$ is a continuous function of $\omega$, analytic as a function of $\beta$ and

$$
\begin{aligned}
& \left.\frac{\partial}{\partial \beta} J(\beta, \omega)\right|_{\beta=0}=I(\omega), \\
& J(\beta, \omega)=\frac{\beta}{8}\left(\frac{\beta}{2}+1\right) \tau^{2} \omega^{2}+0\left(\omega^{3-\delta}\right), \omega \downarrow 0,1>\delta>0 . \\
& J(\beta, \omega) \text { is convex as a function of } \beta .
\end{aligned}
$$

Proof of Corollary 1. From (3.5) and (3.11) we get

$$
\begin{aligned}
\left\langle T^{*} T(N, \omega)\right\rangle & \leqq 4\left\langle\left\{2+\alpha_{1} e^{2 N \eta_{N}}\right\}^{-1}\right\rangle \leqq 2 P\left(\eta_{N} \leqq I(1-\varepsilon)\right)+\frac{4}{\alpha_{1}} e^{-2 N I(1-\varepsilon)} \\
& \leqq 2 K e^{\beta N I(1-\varepsilon)+J(-\beta, \omega) N}+\frac{4}{\alpha_{1}} e^{-2 N I(1-\varepsilon)} \\
& \leqq 2 \max \left(2 K, \frac{4}{\alpha_{1}}\right) e^{N \max \{\beta I(1-\varepsilon)+J(-\beta, \omega),-2 I(1-\varepsilon)\}}
\end{aligned}
$$

By (3.12) and the continuity of $J$ and $I$, it is possible for fixed $1>\varepsilon>0$ to find $\beta_{0}(\omega)$ $>0$ such that $\beta_{0} I(1-\varepsilon)+J\left(-\beta_{0}, \omega\right)<0$ and is a continuous function of $\omega$ for $\omega>0$. This with (3.14) gives us Corollary 1 except for the small $\omega$ behavior of $g(\omega)$. In order to obtain the small $\omega$ behavior, we have to optimize the inequality (3.14) by choosing $\beta$ and $\varepsilon$ optimal. This is a simple calculation using the small $\omega$ behavior 
of $J$ and $I$ from (3.8) and (3.13). The choice $\beta=\varepsilon=\sqrt{8}-2$ is optimal and gives $\lim _{\omega \downarrow 0} g(\omega) / \omega^{2}=\left(\frac{3}{4}-\frac{1}{2} \sqrt{2}\right) \tau^{2}=g_{0}$.

Proof of Corollary 2. First we rewrite (3.6) such that $r_{N}$ and $q_{N}$ appear in separate factors

$$
\begin{aligned}
T^{*} T(N, \omega) & \geqq 4\left\{2+\alpha_{2} r_{N}^{2}+\alpha_{2} q_{N}^{2}\right\}^{-1} \geqq 8\left\{2+\alpha_{2} r_{N}^{2}\right\}^{-1}\left\{2+\alpha_{2} q_{N}^{2}\right\}^{-1} \\
& =8 e^{-\log \left(2+\alpha_{2} r_{N}^{2}\right)-\log \left(2+\alpha_{2} q_{N}^{2}\right)} .
\end{aligned}
$$

By Jensen's inequality

$$
\begin{aligned}
\left\langle T^{*} T(N, \omega)\right\rangle & \geqq 8 e^{-\left\langle\log \left(2+\alpha_{2} r_{N}^{2}\right)\right\rangle-\left\langle\log \left(2+\alpha_{2} q_{N}^{2}\right)\right\rangle} \\
& \left.=8 e^{-N\left\{\frac{1}{N}\left\langle\log \left(2+\alpha_{2} r_{N}^{2}\right)\right\rangle+\frac{1}{N}\left\langle\log \left(2+\alpha_{2} q_{N}^{2}\right)\right\rangle\right.}\right\} .
\end{aligned}
$$

Next we construct upperbounds for the two terms in the exponent using the large deviation estimates (3.9) and (3.13). Since the proofs of both bounds are completely analogous, we prove only one. By splitting the expectation integral we get

$$
\begin{aligned}
\frac{1}{N}\left\langle\log \left(2+\alpha_{2} e^{2 N \varrho_{N}}\right)\right\rangle \leqq & \frac{1}{N} \log \left(2+\alpha_{2} e^{2 N I(1+\varepsilon)}\right) P\left(\varrho_{N} \leqq I(1+\varepsilon)\right) \\
& +\sum_{j=1}^{\infty} \frac{1}{N} \log \left(2+\alpha_{2} e^{2 N I(1+\varepsilon+j \varepsilon)}\right) P\left(I(1+j \varepsilon)<\varrho_{N}\right. \\
& \leqq I(1+\varepsilon+j \varepsilon)) .
\end{aligned}
$$

Since $\alpha_{2}>\sqrt{2}$, as is immediate from (3.6), and since $I$ is positive

$$
\log \left(2+\alpha_{2} e^{2 N I(1+j \varepsilon)}\right) \leqq \log \left(\alpha_{2}+\alpha_{2} \sqrt{2}\right)+2 N I(1+j \varepsilon) .
$$

Substituting this inequality and rearranging the terms we find

$$
\begin{aligned}
\frac{1}{N}\left\langle\log \left(2+\alpha_{2} \mathrm{e}^{2 N \varrho_{N}}\right)\right\rangle \leqq & \frac{1}{N} \log \left(\alpha_{2}+\alpha_{2} \sqrt{2}\right)+2 I(1+\varepsilon) \\
& +2 \varepsilon I \sum_{j=1}^{\infty} P\left(\varrho_{N}>I(1+j \varepsilon)\right)
\end{aligned}
$$

and by (3.9)

$$
\begin{aligned}
\frac{1}{N}\left\langle\log \left(2+\alpha_{2} e^{2 N \varrho_{N}}\right)\right\rangle \leqq & \frac{1}{N} \log \left(\alpha_{2}+\alpha_{2} \sqrt{2}\right)+2 I(1+\varepsilon) \\
& +2 \varepsilon I K \frac{e^{N\{J(\beta, \omega)-\beta I(1+\varepsilon)\}}}{1-e^{-\beta N I \varepsilon}} .
\end{aligned}
$$

We have the same estimate for $\eta_{N}$. Substituting these estimates in (3.15) we obtain for $0<\omega<\omega_{0}$

$$
\left\langle T^{*} T(N, \omega)\right\rangle \geqq \frac{8}{\alpha_{2}^{2}(1+\sqrt{2})^{2}} \exp \left\{-2 N\left[2 I(1+\varepsilon)+2 \varepsilon I K \frac{e^{N\{J(\beta, \omega)-\beta I(1+\varepsilon)\}}}{1-e^{-N \beta I \varepsilon}}\right]\right\} .
$$


By the small $\omega$ behavior of $I$ and $J$ we find

$$
\lim _{N \rightarrow \infty}\left\langle T^{*} T\left(N, \frac{\omega}{\sqrt{N}}\right)\right\rangle \geqq \frac{8}{\alpha_{2}^{2}(1+\sqrt{2})^{2}} \exp -\left[(1+\varepsilon) \frac{\tau^{2} \omega^{2}}{2}+\frac{\varepsilon K \tau^{2} \omega^{2}}{2} \frac{e^{\frac{\beta^{2} \tau^{2}}{16} \omega^{2}}}{e^{\beta \varepsilon \frac{\tau^{2} \omega^{2}}{8}}-1}\right]
$$

Of course we can optimize this inequality, but to obtain Corollary 2 this is sufficient.

\section{Proof of Theorem 2}

From the definition of the variables $\phi_{j}, \theta_{j}, r_{j}, q_{j}$ and the dynamic Eq. (1.1) it is easily seen that

$$
\phi_{n+1}=2 \arctan \frac{\left(2-m_{n} \omega^{2}\right) \frac{\langle m\rangle^{1 / 2} \omega}{2} \tan \frac{\phi_{n}}{2}-m_{n} \omega^{2}}{\frac{\langle m\rangle^{1 / 2} \omega}{2}\left(2-m_{n} \omega^{2}\right)+\frac{\langle m\rangle \omega^{2}}{4}\left(4-m_{n} \omega^{2}\right) \tan \frac{\phi_{n}}{2}}=\psi\left(\phi_{n}, m_{n}\right)
$$

and

$$
\begin{aligned}
r_{n+1}= & \frac{r_{n}}{\langle m\rangle^{1 / 2} \omega}\left\{\left[\frac{\langle m\rangle^{1 / 2} \omega}{2}\left(2-m_{n} \omega^{2}\right) \cos \frac{\phi_{n}}{2}+\frac{\langle m\rangle \omega^{2}}{4}\left(4-m_{n} \omega^{2}\right) \sin \frac{\phi_{n}}{2}\right]^{2}\right. \\
& \left.+\left[\frac{\langle m\rangle^{1 / 2} \omega}{2}\left(2-m_{n} \omega^{2}\right) \sin \frac{\phi_{n}}{2}-m_{n} \omega^{2} \cos \frac{\phi_{n}}{2}\right]^{2}\right\}^{1 / 2} \\
= & r_{n} \chi\left(\phi_{n}, m_{n}\right) .
\end{aligned}
$$

We have the same relations for $\theta_{j}$ and $q_{j}$. By the symmetry of (4.1) and (4.2) we can now consider $0 \leqq \phi_{n}<2 \pi, 0 \leqq \theta_{n}<2 \pi$, i.e., we are no longer interested in the distinction between $\left(w_{j+1}, w_{j}\right)=(a, b)$ and $\left(w_{j+1}, w_{j}\right)=(-a,-b)$. We notice that since the factor by which $r_{n}$ is multiplied to get $r_{n+1}$ is bounded above and below by positive constants independent of $m_{n}$ and $\phi_{n}$, we have that $\varrho_{n}$, given $r_{0}$, is uniformly bounded, with the bounds independent of $n, \phi_{0}$, and $m_{j}$. Also we notice that $\psi(\phi, m)$ is a monotonic function of $\phi$ and $m$, as a function of $\phi 1-1$ onto. Now it is clear that the random sequence $\left\{\phi_{j}\right\}$ is a Markov chain with transition probability

$$
P(\phi, \Phi)=\int P_{m}(d m) \mathrm{X}_{\Phi}(\psi(\phi, m))
$$

where $\Phi$ is an arbitrary Borel set of the unit circle, and $X_{\Phi}$ is its characteristic function. In the sequel we will denote the usual Lebesgue measure on the unit circle by $v(\cdot)$.

First we prove that Doeblin's condition $D$ (cf. Doob [14]) holds, which gives us the ergodic properties of the Markov chain. (See O'Connor [5]).

Lemma 1. i) There exists an $\varepsilon>0$ such that $P^{2}(\phi, \Phi) \leqq 1-\varepsilon$ whenever $v(\Phi) \leqq \varepsilon$

ii) There is only one ergodic set, the transition set has measure zero, there are no cyclically moving subsets for $0<\omega<\omega_{0}$. 
This implies

iii) There exist a unique limiting distribution $\bar{P}$, and constants $\gamma$ and $\varrho<1$ such that

$$
\left|P^{n}(\phi, \Phi)-\bar{P}(\Phi)\right| \leqq \gamma \varrho^{n} \text { for all } \phi \text { and } \Phi .
$$

iv) $\bar{P}(\Phi)$ is positive whenever $v(\Phi)$ is positive.

Proof. We have $P^{2}(\phi, \Phi)=\iint d m d m^{\prime} \mu(m) \mu\left(m^{\prime}\right) \mathbf{X}_{\Phi}\left(\psi\left(\psi\left(\phi, m^{\prime}\right), m\right)\right)$.

Now consider $\psi\left(\psi\left(\phi, m^{\prime}\right), m\right)$ as a function of $m^{\prime}$ and $m$. Then it is easily shown that the 2-dimensional gradient of this function has a positive lower bound $l$ independent of $\phi$. So the Lebesgue measure of the set in the $\left(\mathrm{m}^{\prime}, m\right)$-plane that contributes to the integral (4.4), which is

$$
\lambda(\Phi)=\iint \mathbf{X}_{\Phi}\left(\psi\left(\psi\left(\phi, m^{\prime}\right), m\right)\right) d m^{\prime} d m,
$$

is bounded by

$$
2 \frac{\left(m^{(1)}-m^{(0)}\right)}{l} v(\Phi) .
$$

Next we invoke a well-known theorem from integration theory, see, e.g., Gikhman, Skorokhod [15, p. 71]. This gives us Doeblin's condition D. For the proof that there is only one ergodic set, the whole set, and for the absence of cyclically moving subsets we refer to O'Connor [5].

The conclusions (iii) and (iv) are now immediate from Doob [14].

Now we follow Pastur and Feldman, and consider

$$
\left\langle e^{\beta N \varrho_{N}}\right\rangle \text { and }\left\langle e^{\beta N \eta_{N}}\right\rangle, \quad \beta \in \mathbb{R} \text {. }
$$

Then it is easily seen that we have

$$
\begin{array}{ll}
P\left(\varrho_{N}>I(1+\varepsilon)\right) \leqq e^{-\beta N I(1+\varepsilon)}\left\langle e^{\beta N \varrho_{N}}\right\rangle, & \beta \geqq 0 . \\
P\left(\eta_{N} \leqq I(1-\varepsilon)\right) \leqq e^{-\beta N I(1-\varepsilon)}\left\langle e^{\beta N \eta_{N}}\right\rangle, & \beta \leqq 0 .
\end{array}
$$

The next step must be an estimate of the expressions (4.5). For that we introduce the following operator, defined on the space of continuous functions on the unit circle with supnorm.

$$
A f(\phi)=\int P_{m}(d m) \chi^{\beta}(\phi, m) f(\psi(\phi, m))
$$

where $\psi(\phi, m)$ is defined by (4.1) and $\chi(\phi, m)$ by (4.2).

The dependence of $A$ on $\beta$ and $\omega$ will usually not be indicated. Now it is easily checked that

$$
A^{n} f(\phi)=\dot{E}_{\phi}\left(e^{\beta n \varrho_{n}} f\left(\phi_{n}\right)\right)
$$

where $E_{\phi}$ stands for the conditional expectation given $\phi_{0}=\phi$, and $\varrho_{n}$ is defined by (4.2) as a function of the phase variables by taking $r_{0}=1$. Thus

$$
\begin{aligned}
& \left\langle e^{\beta \varrho_{N}}\right\rangle=A^{N} 1(0), \\
& \left\langle e^{\beta N \eta_{N}}\right\rangle=A^{N} 1(\pi),
\end{aligned}
$$


where 1 stands for the function identically 1 . To construct estimates we must have information about the spectrum of $A$.

Lemma 2. i) The operator $A$ is positivity preserving, and moreover strongly positive, i.e., for every $f \geqq 0, f \neq 0$, there is an $m$ such that $A^{m} f$ is strictly positive. The strong positivity we have for $0<\omega<\omega_{0}$.

ii) $A^{2}$ is compact.

iii) $A^{2}$ depends continuously on $\omega$ (in the norm sense) for $\omega>0, A$ depends analytically on $\beta$.

Proof. i) Positivity. The positivity preserving property is trivial. Since $\chi \geqq \chi_{0}>0$ we have

$$
A^{n} f(\phi) \geqq \chi_{0}^{n \beta} \int P^{n}(\phi, d \phi) f(\phi) .
$$

Take $f \geqq 0, f \neq 0$. Let $\Phi$ be such that $f(\phi) \geqq \delta>0$ for $\phi \in \Phi$. Then

$$
A^{n} f(\phi) \geqq \delta \chi_{0}^{n \beta} P^{n}(\phi, \Phi) .
$$

Next we use Lemma 1 (iv) saying that $\bar{P}(\Phi)$ is positive. So by Lemma 1 (iii) for sufficiently large $n P^{n}(\phi, \Phi)$ is positive, which proves the strong positivity.

ii) Compactness. We use an idea due to O'Connor [5]. Since, however, we could not verify all the steps in his proof, we will give a proof here. Because the continuous functions are dense in $L^{1}$ we can approximate the operator $A$ in norm by operators with a continuous density $\mu$. We prove that $A^{2}$ with continuous $\mu$ is compact. In (4.8) we use $\psi$ as integration variable instead of $m$, with $m=m(\psi, \phi)$ :

$$
A f(\phi)=\int d \psi \frac{\partial m}{\partial \psi} \mu(m(\psi, \phi)) \chi^{\beta}(\phi, m(\psi, \phi)) f(\psi)
$$

Now it is easily checked that $\frac{\partial \psi}{\partial m}$ is bounded away from zero if

$$
\left|\tan \frac{\phi}{2}+\frac{2}{\langle m\rangle^{1 / 2} \omega}\right|>\varepsilon, \quad \varepsilon>0 .
$$

So we define two continuous functions $g_{1}(\phi)$ and $g_{2}(\phi)$ such that

$$
g_{1}(\phi)+g_{2}(\phi)=1
$$

and such that

$$
\begin{aligned}
& g_{1}(\phi)=0 \text { if }\left|\tan \frac{\phi}{2}+\frac{2}{\langle m\rangle^{1 / 2} \omega}\right|<\varepsilon \\
& g_{2}(\phi)=0 \text { if }\left|\tan \frac{\phi}{2}+\frac{2}{\langle m\rangle^{1 / 2} \omega}\right|>2 \varepsilon .
\end{aligned}
$$

Then by the Arzela-Ascoli theorem for any bounded family $f_{n}$ the family $g_{1} A f_{n}$ contains a convergent subsequence, and, of course, $A g_{1} A f_{n}$ contains also a convergent subsequence. Next we consider $A g_{2} A f_{n}=A g_{2} f_{n}^{\prime}$ and show that this family contains a convergent subsequence.

$$
A g_{2} f_{n}^{\prime}(\phi)=\int d m \mu(m) \chi^{\beta}(\phi, m) g_{2}(\psi(\phi, m)) f_{n}^{\prime}(\psi(\phi, m)) \text {. }
$$


Next we change variables again. Now we avoid the dangerous neighborhood where $\left|\tan \frac{\phi}{2}+\frac{2}{\langle m\rangle^{1 / 2} \omega}\right|<\delta$, since there $g_{2}(\psi(\phi, m))=0$ as is easily checked. (We note that this procedure is closely related to the procedure we used to prove Doeblin's condition.)

iii) Continuity Properties. The dependence on $\beta$ is trivial. By a procedure similar to the one we used to prove compactness, we prove that $A^{2}$ depends continuously in norm on $\omega$ for $\omega>0$. (If we would not have introduced an $\omega$ in the definitions (3.2-3), $A$ itself would depend continuously on $\omega$, as could be shown by a change of variables $m^{\prime}=m \omega^{2}$.)

Now we are in the position to use the Krein-Rutman theorem [16]: $A^{2}$ has a unique strictly positive eigenvector with positive eigenvalue which exceeds all other eigenvalues in modulus. We denote the eigenvalue by $e^{2 J}$ and the eigenfunction by $f_{0}(\phi)$. Due to the continuity properties of $A$ and the fact that the eigenvalue is isolated, we have that $J(\beta, \omega)$ depends continuously on $\omega$, and so does $f_{0}$ (in the norm sense) for $\omega>0 . J$ is analytic as a function of $\beta$ and so is $f_{0}$. So we have for $\omega>\omega_{1}>0$

$$
A^{N} 1(\phi) \leqq A^{N} \frac{f_{0}}{\min _{\phi} f_{0}}=e^{N J(\beta, \omega)} \frac{f_{0}}{\min _{\phi} f_{0}} \leqq K e^{N J(\beta, \omega)} .
$$

This with (4.6) and (4.7) gives us (3.9), (3.10), and (3.11). Next we prove that

$$
\left.\frac{\partial}{\partial \beta} J(\beta, \omega)\right|_{\beta=0}=I(\omega) \text {. }
$$

We use the following representation of $J$

$$
J(\beta, \omega)=\lim _{n \rightarrow \infty} \frac{1}{n} \log A^{n} f_{0} .
$$

Since $J(0, \omega)=0$ we must prove that

$$
\lim _{\beta \rightarrow 0} \lim _{n \rightarrow \infty} \frac{1}{n \beta} \log A^{n} f_{0} \approx I \text {. }
$$

Since $f_{0}$ depends analytically on $\beta$ the order of the limits is not important. Next we use the representation (4.9)

$$
A^{n} f_{0}(\phi)=E_{\phi}\left(e^{\beta n \varrho_{n}} f_{0}\left(\phi_{n}\right)\right) \text {. }
$$

Since for fixed $n, n \varrho_{n}$ is uniformly bounded, and $f_{0}(\omega=0) \equiv 1$, we find by first taking the limit $\beta \rightarrow 0$ and next $n \rightarrow \infty$

$$
\lim _{n \rightarrow \infty} \lim _{\beta \rightarrow 0} \frac{1}{n \beta} \log A^{n} f_{0}=\lim _{n \rightarrow \infty}\left\langle\varrho_{n}\right\rangle=I
$$

by Theorem 1 .

The only part of Theorem 2 that remains to be proven, is the small $\omega$ behavior of $J(\beta, \omega)$. We do this by a perturbation expansion. The proof of this expansion is the reason for the introduction of the factors $\frac{\langle m\rangle^{1 / 2} \omega}{2}$ in $(3.2-3)$. 
Without the factor $\omega$ the problem would not be regular, and without the $\langle m\rangle^{1 / 2}$ only solvable for $\langle m\rangle=1$.

By doing the perturbation expansion in a formal way we find

$$
\begin{aligned}
f_{0}(\phi) & =1+\omega \frac{\tau^{2}}{\langle m\rangle^{1 / 2}}\left\{\frac{\beta}{8} \sin \phi-\frac{\beta}{32}\left(\frac{\beta}{2}-1\right) \sin 2 \phi\right\}+\ldots \\
& =1+\omega h(\phi)+\ldots
\end{aligned}
$$

and

$$
e^{J(\beta, \omega)}=1+\omega^{2} \frac{\beta}{8}\left(\frac{\beta}{2}+1\right) \tau^{2}+\ldots
$$

Now consider

$$
f(\phi)=1+\omega h(\phi) .
$$

Then constants $f_{-}$and $f_{+}$exist such that $\infty>f_{+} \geqq f_{-}>0$ for $\omega$ sufficiently small. So

$$
1 \leqq \frac{f}{f_{-}}
$$

and

$$
A^{n} 1 \leqq \frac{1}{f_{-}} A^{n} f .
$$

Next it is not difficult to verify that

$$
A f=e^{\omega^{2} \frac{\beta}{8}\left(\frac{\beta}{2}+1\right) \tau^{2}} f e^{0\left(\omega^{3}\right)}
$$

therefore

$$
e^{-\omega^{2} \frac{\beta}{8}\left(\frac{\beta}{2}+1\right) \tau^{2}-\gamma \omega^{3-\delta}} A f=f e^{0\left(\omega^{3}\right)-\gamma \omega^{3-\delta}} \leqq f
$$

for $\gamma>0, \delta>0$, and $\omega<\omega_{2}, \omega_{2}$ sufficiently small.

Induction then gives

$$
e^{-n \omega^{2} \frac{\beta}{8}\left(\frac{\beta}{2}+1\right) \tau^{2}-n \gamma \omega^{3-\delta}} A^{n} f \leqq f \leqq f_{+}
$$

and

$$
A^{n} 1 \leqq \frac{f_{+}}{f_{-}} e^{n \omega^{2} \frac{\beta}{8}\left(\frac{\beta}{2}+1\right) \tau^{2+n \gamma \omega^{3-\delta}}} .
$$

This together with (4.6), (4.7) and (4.10), (4.11) gives us the small $\omega$ part of Theorem 2 and the proof is complete.

\section{Discussion}

The asymptotic behavior of products of random matrices, which is the general framework in which the present work best fits, is a subject currently of great 
interest in a variety of fields ranging from studies of spectra of random systems [8], [19], to finding models for turbulence [20]. We will discuss here briefly our work in this context. Let $M=\left(m_{0}, m_{1}, \ldots\right)$ be a point in $\Omega$, the space of semi-infinite sequences with $m_{j} \in \mathbb{R}_{+}$. We put on $\Omega$ the product measure of $P_{m}(d m)$ and ask for the asymptotic behavior of the random variable, the two by two matrix

$$
S^{N}(M)=\prod_{j=0}^{N-1} S_{j}(M)
$$

or of the norm $\left\|S^{N}(M) u\right\|$ where $u$ is a real non-zero vector. We have specifically that

$$
S_{j}(M)=S\left(m_{j}\right)=K-\lambda x_{j} U \in \operatorname{SL}(2)
$$

Here $\lambda=\omega^{2} \sigma$ and $x_{j}=\frac{m_{j}}{\sigma}$, a random variable with unit dispersion. $K$ is the fixed matrix

$$
K=\left(\begin{array}{rr}
2 & -1 \\
1 & 0
\end{array}\right)
$$

and

$$
U=\left(\begin{array}{ll}
1 & 0 \\
0 & 0
\end{array}\right) .
$$

To obtain our results about the heat conductivity we needed and were able to prove (or find in the literature) the results stated in Theorem 1 and 2 of Sect. 3 about

$$
\varrho_{N}=\frac{1}{N} \ln \left\|S^{N} u\right\|
$$

for two particular choices of $u$. The results are actually independent of the specific choice of $u$. But they do depend on the following facts. For Theorem 1 we need (i) $\operatorname{det} S\left(m_{j}\right)=1$ (ii) certain group theoretic properties which for the form given in (5.1) are satisfied whenever the support of $P_{m}(\mathrm{dm})$ contains at least two points (possibly negative) [12], [13]. For Theorem 2 we used Theorem 1 and needed more information about $P_{m}(d m)$. In particular important information was obtained about the behavior of $\varrho_{N}$ for small values of $\omega$ and about large deviations.

The question is: how much of Theorem 1 and 2 really depends on the specific assumptions, i.e., how much would remain valid for more general matrices $K$ and $U$ (not necessarily two by two). This would be important if we want to apply our methods to other problems like the heat conduction in a strip which can also be treated by the transfer matrix approach.

Acknowledgements. The author thanks Prof. J. L. Lebowitz for his hospitality and many enlightening discussions. He also thanks Prof. G. C. Papanicolau for helpful discussions and making available the manuscript of Ref. [6] prior to publication. 


\section{References}

1. Casher, A., Lebowitz, J.L.: J. Math. Phys. 12, 1701 (1971)

2. Rubin, R.J., Greer, W.L.: J. Math. Phys. 12, 1686 (1971)

3. O'Connor, A.J., Lebowitz, J.L. : J. Math. Phys. 15, 692 (1974)

4. Pastur, L.A., Feldman, E.P.: Sov. Phys. JETP 40, 241 (1975)

5. O'Connor, A.J.: Commun. Math. Phys. 45, 63 (1975)

6. Papanicolau, G.C.: On the instability of the random harmonic oscillator. Preprint (1978)

7. Spohn, H., Lebowitz, J.L.: Commun. Math. Phys. 54, 97 (1977)

8. Matsuda, M., Ishii, K.: Suppl. Prog. Theor. Phys. 45, 56 (1970)

9. Payton, D.N., Rich, M., Visscher, W.M. : Phys. Rev. 160, 706 (1967)

10. Keller, J.B., Papanicolau, G.C., Weilenmann, J. : Commun. Pure Appl. Math. 31, 583 (1978)

11. Papanocilau, G.C.: Unpublished notes (1976)

12. Yoshioka, Y.: Proc. Jpn. Acad. 49, 665 (1973)

13. Furstenberg, H.: Trans. A.M.S. 108, 377 (1963)

14. Doob, J.L.: Stochastic processes. New York: Wiley 1953

15. Gikhman, I.I., Skorokhod, A.V.: Introduction to the theory of random processes. Philadelphia, London, Toronto: Saunders 1969

16. Krein, M.G., Rutman, M.A. : Usp. Mat. Nauk. 23, 3 (1948) (A.M.S Transl. no. 26 (1950))

17. Visscher, W.M. : Prog. Theor. Phys. 46, 729 (1971)

18. Visscher, W.M.: Methods Comput. Phys. 15, 371 (1976)

19. Goldshtein, I.Ya, Molchanov, S.A., Pastur, L.A.: Funct. Anal. Appl. 11, 1 (1977)

20. Ruelle, D.: Ann. N.Y. Acad. Sci. Conf. on Bifurcation (1978). In press

\section{Communicated by J. L. Lebowitz}

Received March 16, 1979 